\title{
Oportunidades de aprendizaje en matemáticas para estudiantes con discapacidad intelectual
}

Math Learning Opportunities for Students with Learning Disabilities

Oportunidades de aprendizagem das matemáticas para estudantes com deficiência intelectual

\author{
Sebastián Howard-Montaner*† iD orcid.org/0000-0002-3621-5738 \\ Constanza San Martín** iD orcid.org/0000-0001-5948-1329 \\ Natalia Salas-Guzmán*** (iD) orcid.org/0000-0001-8815-5979 \\ Pamela Margarita Blanco-Vargas**** (D) orcid.org/0000-0002-2049-38xxx \\ Cecilia Jacqueline Díaz-Cárcamo***** iD orcid.org/0000-0002-2999-880x
}

\section{Artículo de investigación \\ Revista Colombiana de Educación, N. 74 . Primer semestre de 2018, Bogotá, Colombia \\ Para citar este artículo: Howard, S., San Martin, C., Salas, N., Blanco, P., y Díaz, C. (2O18). \\ Oportunidades de aprendizaje en matemáticas para estudiantes con discapacidad intelectual \\ Revista Colombiana de Educación, (74), 197-219.}

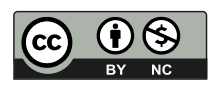

Recibido: 30/01/2017

Evaluado: 19/02/2017

* El profesor Sebastián Howard, primer autor del presente artículo, murío el pasado 27 de agosto. La Revista Colombiana de Educación rinde un homenaje a su trayectoria y expresa sus condolencias a su familia y amigos.

Doctor en Ciencias de la Educación. Profesor asistente de la Facultad de Educación de la Universidad Diego Portales. Santiago de Chile, Chile.

** Doctora en Psicología Escolar y Desarrollo. Profesora asistente de la Facultad de Educación de la Univer sidad Diego Portales. Santiago de Chile, Chile. Correo electrónico: constanza.sanmartin@amail.udp.cl

*** Doctora en Psicología, Pontificia Universidad Católica de Chile. Profesora asociada de la Facultad de Edu cación de la Universidad Diego Portales, Santiago de Chile. Correo electrónico: natalia.salas@mail.udp.cl

**** Magíster en Educación. Profesora de la Universidad Santo Tomás. Santiago de Chile, Chile. Correo electrónico: blancopam(a) gmail.com

***** Magíster en Literatura, Teoría. Profesora de Lenguaje, Instituto de las Religiosas de la Inmaculada Concepción, Chile. Correo electrónico: ceciliajdiazcarcamo@gmail.com 


\section{Resumen}

En Chile, los estudiantes con discapacidad intelectual (DI) asisten a escuelas especiales o a escuelas regulares con programas de integración escolar (PIE). Hasta la fecha se carece de información respecto de las oportunidades de aprendizaje (ODA) que se proporcionan a estos estudiantes en ambos contextos. El estudio que se presenta es parte de una investigación mayor, cuyo propósito es explorar las ODA de las matemáticas que se generan en centros de educación especial para estudiantes con DI. Se realizaron entrevistas semiestructuradas con profesores y directivos de escuelas especiales, con el objetivo de indagar cuáles son sus creencias sobre la enseñanza, sobre los estudiantes con DI y las matemáticas. Los resultados indican que la enseñanza a estudiantes con DI requiere de mucho tiempo de planificación, de un currículo flexible que se adapte a las necesidades individuales, de uso de material concreto y de una evaluación individualizada. Respecto de los estudiantes, los entrevistados indican que no hay fórmulas generales y que se debe tratar cada caso de manera individual. Finalmente, profesores y directivos estiman que la enseñanza de las matemáticas para estudiantes con Di son principalmente el conteo y las operaciones con números, lo cual excluye del currículo ofrecido espacios para el desarrollo de otras habilidades matemáticas.

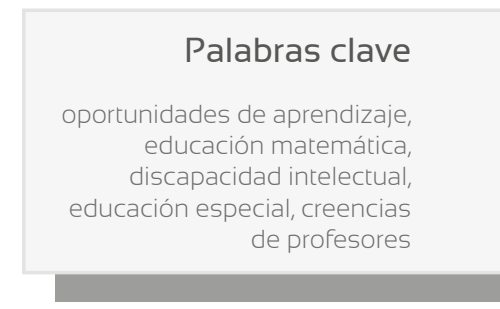

\section{Keywords}

learning opportunities; math education; learning disabilities; special education; teacher beliefs

\begin{abstract}
In Chile, students with learning disabilities (LD) attend either special schools or regular schools with school integration programs (SIP). To this day, there is no information about the learning opportunities (LO) provided to these students in both contexts. The study is part of a larger research aimed at exploring the mathematics Lo for students with LD in special education centers. We conducted semi-structured interviews with teachers and principals from special schools with the purpose of learning about their beliefs on teaching, students with LD, and math. Results show that teaching students with LD requires many hours of planning, a flexible curriculum adapted to their individual needs, using specific material, and individualized assessment. Regarding students, the respondents note that there are no general formulas and that each case should be handled individually. Finally, the school teachers and principals estimate that the teaching of mathematics for students with LD includes mainly counting and operations with numbers, which excludes spaces for developing other mathematical abilities from the curriculum offered.
\end{abstract}

\section{Resumo}

No Chile, os estudantes com deficiência intelectual (DI) frequentam escolas especiais ou escolas normais com programas de integração escolar (PIE). Até agora não existem dados sobre as oportunidades de aprendizagem (ODA) proporcionadas aos estudantes nos dois contextos. O estudo apresentado faz parte de uma pesquisa maior, cujo propósito é explorar as ODA das matemáticas que são geradas em centros de educação especial para estudantes com DI. Realizaram-se entrevistas semiestruturadas com professores e diretivos de escolas especiais, com o objetivo de indagar quais são suas crenças sobre o ensino, sobre os estudantes com DI e as matemáticas. Os resultados assinalam que o ensino a estudantes com DI precisa de muito tempo de planejamento, de um currículo flexível que possa ser adaptado às necessidades individuas, de uso de material concreto e de uma avaliação individualizada. Em relação com os estudantes, os entrevistados indicam que não existem fórmulas gerais e que cada caso deve ser trabalhado individualmente. Finalmente, professores e diretivos consideram que o ensino das matemáticas para estudantes com DI consiste principalmente na contagem e as operações com números, o que exclui do currículo oferecido espaços para o desenvolvimento de outras habilidades matemáticas.

\section{Palavras-chave}

oportunidades de aprendizagem; educação matemática; deficiência intelectual; educação especial; crenças de professores 


\section{Introducción}

Uno de los mayores problemas para la teoría y la práctica de la educación matemática es la equidad (Forgasz y Rivera, 2012). Especialmente si entendemos que esta no puede ocurrir sin el acceso a la educación matemática (Bishop y Forgasz, 2007). Es por ello que cobra relevancia, en los estudios de equidad en educación matemática, indagar cómo acceden a los objetivos de aprendizaje del currículo todos los estudiantes y, de un modo particular, aquellos que por condiciones personales o contextuales se encuentran en riesgo de exclusión, como es el caso de personas en condición de discapacidad intelectual ${ }^{1}$ (DI) (Ainscow, Booth y Dyson, 2006).

Los objetivos de aprendizaje del currículo chileno están prescritos para todos los estudiantes. Sin embargo, investigaciones a nivel internacional muestran que los estudiantes con DI no suelen tener acceso a programas de matemáticas de alta calidad educativa (Gervasoni y Lindenskov, 2011). Muchos de ellos son excluidos de las oportunidades para aprender matemática porque se considera un campo de estudio inadecuado para ellos (Faragher, Brady, Clarke y Gervasoni, 2008). Más aún, a los estudiantes con DI que han sido parte de experiencias de enseñanza de las matemáticas, la calidad de esta y las ODA proporcionadas no les han permitido prosperar (Gervasoni y Sullivan, 2007). Estos estudiantes han tenido históricamente un currículo menos riguroso con planes educativos individuales y una vinculación mínima con los aprendizajes establecidos en marcos curriculares (Nolet y McLaughlin, 2000).

La naturaleza abstracta y conceptual de las matemáticas plantea retos particulares a los estudiantes con DI, principalmente en resolución de problemas (Cawley y Miller, 1989). Estos retos pueden estar asociados a dificultades en procesos de memoria, en el uso de estrategias metacognitivas (Gallico, Burns y Grob, 1991), en obstáculos para prestar atención a dimensiones clave de la tarea y para transferir aprendizajes (Kauffman, 2001). Por lo tanto, necesitan más oportunidades para utilizar materiales concretos, realizar tareas breves y variadas (Sarama y Clements, 2009).

En Chile los estudiantes con Di asisten a escuelas especiales o a escuelas regulares con programas de integración escolar. Aun cuando se cuenta con datos referidos a su acceso al sistema educativo, poco se conoce respecto a las ODA que se les proporcionan.

1 La DI está definida como dificultad en el funcionamiento en cinco dimensiones: 1) habilidades intelectuales; 2) conducta adaptativa; 3) participación, interacción y roles sociales; 4) salud, y 5) contexto. La evaluación se basa en la Escala de Inteligencia de Wechsler (versión adaptada y estandarizada para Chile en 2005 con un ci $\geq 69$ ), con énfasis en tres criterios asociados al funcionamiento intelectual: presencia de limitaciones significativas del funcionamiento intelectual, limitaciones significativas de la conducta adaptativa y edad de aparición es antes de los 18 años (OMS, 2001). 
En este contexto, la presente investigación tiene por objetivo explorar y describir las ODA en matemáticas que se generan en escuelas especiales para estudiantes que presentan DI. Específicamente, en este trabajo se reporta el resultado del análisis de entrevistas semiestructuradas desarroIladas con profesores y directivos docentes de escuelas especiales chilenas, encaminadas a conocer cuáles son las creencias y prácticas declaradas respecto a las matemáticas y su proceso de enseñanza y aprendizaje con estudiantes que presentan D. Todo ello con miras a comprender la implementación de las ODA en sus aulas.

\section{Antecedentes}

\section{Enseñanza de las matemáticas a estudiantes con DI}

Las matemáticas proporcionan a los estudiantes el lenguaje a través del cual pueden interpretar, describir, analizar, hacer predicciones y solucionar problemas en la vida cotidiana. Esto les permite participar en una amplia gama de experiencias y relaciones matemáticas en la escuela y en la vida diaria. Sin embargo, quienes presentan DI a menudo encuentran dificultades para desarrollar tareas cotidianas (decir/ver la hora, ir de compras, leer horarios, cocinar, medir, entre otras) que requieren habilidades aritméticas básicas (Cheong, 2014).

Históricamente, se ha pensado que las personas con DI tienen un bajo potencial de aprendizaje matemático (Gervasoni y Sullivan, 2007). Sin embargo, estudios han demostrado que las personas con síndrome de Down tienen conocimientos matemáticos que, desde los procedimientos evaluativos estandarizados, a menudo no logran mostrar (Faragher, Brady, Clarke y Gervasoni, 2008). Del mismo modo, estudios internacionales recientes señalan que los estudiantes con DI pueden desarrollar habilidades matemáticas que están alineadas con varios estándares del currículo nacional, incluso sin modificaciones curriculares significativas (Browder, Trela, Courtade, Jimenez, Knight y Flowers, 2012; Jimenez y Staples, 2015; Jimenez, Browder y Courtade, 2008).

Desde una perspectiva inclusiva, el enfoque actual de la enseñanza de matemáticas para toda población está en el logro de los aprendizajes del currículo nacional de referencia (Jimenez y Staples, 2015). Sin embargo, para muchos estudiantes con DI el acceso a los estándares curriculares generales puede estar limitado por la falta de habilidades numéricas que actúan como requisitos previos (Kearns, Towles-Reeves, Kleinert, Kleinert y Thomas, 2011). El desarrollo de habilidades numéricas o aritméticas tempranas es un predictivo del "éxito" matemático en años posteriores (Sarama y Clements, 2009). Muchos estudiantes con DI pueden no tener 
desarrolladas estas habilidades debido una lenta progresión del desarrollo o a la falta de experiencias y de oportunidades en su proceso educativo (Sarama y Clements, 2009).

La visión de la sociedad y la finalidad de la educación de un país se proyectan en el currículo nacional, por medio de la selección de las competencias necesarias para que sus ciudadanos se desarrollen y participen en los distintos ámbitos de la vida (Duk y Loren, 2010). No obstante, además del marco curricular como referente, es fundamental que se cuente con mecanismos de adecuación y flexibilidad que permitan desarrollar procesos educativos diversificados y pertinentes a las características de los estudiantes. En este sentido, desde el paradigma inclusivo, diferentes autores plantean que la creación de contextos y procesos de aprendizaje debe ser guiada por un currículo común comprensivo para todos los alumnos, mediante una flexibilización curricular (Agut, 2010; Duk y Loren, 2010).

Lo anterior implica necesariamente una enseñanza adaptativa, entendida como "la diversificación de los procesos de enseñanza que permite que todos los estudiantes alcancen los objetivos que el currículum establece" (Martín y Mauri, 1996, p. 14). Así, el ajuste y la flexibilización de los métodos de enseñanza se plantea como principio orientador de la respuesta educativa a la diversidad (Ainscow, 2001). Además, se asume que dentro del aula puede haber alumnos con distintos estilos y niveles de aprendizaje, ante lo cual es necesario realizar adecuaciones curriculares individuales (Stainback y Stainback, 1999).

Estas adecuaciones o adaptaciones curriculares constituyen "un proceso de toma de decisiones compartido tendiente a ajustar y complementar el currículo común para dar respuesta a las necesidades y características de los alumnos y lograr su máximo desarrollo personal y social" (Blanco, 1999, p. 420). La adaptación curricular implica realizar ajustes razonables al currículo prescrito en función de la pertinencia y relevancia para el estudiante en las distintas asignaturas Así, se constituye una herramienta pedagógica que permite equiparar las condiciones para que los estudiantes puedan acceder, participar y progresar en su proceso de enseñanza-aprendizaje $y$, de este modo, contar con oportunidades para desarrollar su aprendizaje a partir de los principios del derecho, la igualdad y la equidad (Duk y Loren, 2010).

\section{Oportunidades de aprendizaje para todos los estudiantes}

Una forma de examinar el acceso que tienen los estudiantes a los aprendizajes que se les entregan, es a través de las ODA. Ellas se definieron originalmente como una medida respecto de la posibilidad de los estudiantes de estudiar un tema en particular o aprender a resolver un determinado tipo de problema presentado en una posterior prueba o evaluación 
(McDonnell, 1995). Posteriormente, se han ampliado hacia la provisión de oportunidades apropiadas para todos los grupos de estudiantes, incluyendo el análisis de los recursos, de las condiciones de la escuela, del currículo y de las experiencias de enseñanza (Banicky, 2000). Este concepto constituye uno de los pocos indicadores que conecta la enseñanza con el aprendizaje (Hiebert y Grouws, 2007; McDonnell, 1995).

Al respecto, Schmidt y McKnight (1995) han planteado que el análisis de las ODA puede dar cuenta de la distancia entre el currículo prescrito y el efectivamente implementado y que las ODA dependen, entre otros factores, de variables asociadas a los profesores, las características de los estudiantes y el ambiente organizacional de la escuela. Según señala Andrew Porter, el currículo implementado involucra las decisiones sobre "cuánto tiempo dedicar a un contenido particular, qué tópicos trabajar, cuándo y en qué orden, en qué nivel de complejidad según las características de unos determinados estudiantes" (Porter, 2002, p. 3).

En el ámbito de la enseñanza de las matemáticas, las ODA han sido entendidas como las acciones que facilitan el aprendizaje y su relación con los aspectos propios del aprendizaje matemático, como señalan Ferrer, Fortuny y Morera (2014). Estos autores identifican las ODA en los procesos de interacción en las aulas de matemáticas.

A nivel internacional los estudios que han buscado explorar las ODA se han Ilevado a cabo por medio de distintas técnicas. Su medición se ha realizado principalmente a través de cuestionarios dirigidos a los profesores (Cogan y Schmidt, 2015; Schmidt y McKnight, 1995), con los cuales se ha buscado conocer el nivel de cobertura curricular y la relación con los aprendizajes de los alumnos, consultando a los docentes en qué medida los estudiantes han sido expuestos a experiencias que les permitan responder a las evaluaciones (McDonnell, 1995; Schmidt et al., 1997).

Otras estrategias que se han utilizado para estudiar las ODA son el análisis de distintos tipos de materiales de aula, como los registros diarios de las actividades realizadas, las planificaciones de clase, las evaluaciones administradas por los profesores (Contreras, 2014) y los materiales didácticos (Boscardin, et al, 2005; McDonnell, 1995). Además, se han realizado estudios por medio del análisis de observaciones de aula, poniendo énfasis en el tipo de tareas matemáticas que los profesores seleccionan y proponen a sus estudiantes, así como el contexto en el que estas se desarrollan (Carrillo, Contreras y Zakaryan, 2014). En estos estudios se ha destacado la relevancia de la actividad del profesor en la generación de ODA, por medio de la selección de tareas y actividades matemáticas (Sullivan, Clarke, Clarke y O'Shea, 2010). En este sentido, un aspecto importante que las investigaciones realizadas hasta la fecha han reconocido es la importancia del profesor y la influencia de sus creencias y percepciones en las ODA 
que se generan para los estudiantes (Sullivan et al., 2010), en particular los objetivos subyacentes a las acciones y toma de decisiones del profesor (Carrillo, Contreras y Zakaryan, 2013).

\section{Creencias de los profesores}

Las creencias están constituidas por un acervo de experiencia, información y emociones. Así, en el desarrollo de las prácticas educativas, los profesores toman decisiones basados no solo en su conocimiento didáctico y disciplinar, sino también en sus convicciones personales respecto al aprendizaje y la enseñanza, entre otros aspectos (Martínez, 2013). En este sentido, ha cobrado interés en el ámbito de la psicología de la educación y del estudio del aprendizaje el análisis de las características de los profesores, específicamente de sus concepciones y creencias, por su influencia en las prácticas pedagógicas (Rodrigo, Rodríguez y Marrero, 1993; San Martín, 2012), en la calidad de la enseñanza y, por lo tanto, de las ODA.

Las creencias matemáticas son componentes del conocimiento subjetivo de los individuos, basados en su experiencia respecto de las matemáticas, su enseñanza y su aprendizaje. Junto con las actitudes y las emociones, constituyen descriptores básicos del dominio afectivo en el aprendizaje de las matemáticas (Gil, Blanco y Guerrero, 2005). En la misma línea, para Gómez-Chacón (2003) las explicaciones a lo que denomina bloqueo en el aprendizaje varían según los sentimientos y actitudes que afectan las estructuras de creencias de los sujetos.

En un artículo reciente acerca de las creencias docentes, Rojas (2014) insiste en la necesidad de avanzar en este campo en Chile, en investigaciones que observen la relación entre lo que creen profesores y profesoras y lo que hacen en sus clases. Pero no desde la perspectiva de subrayar la falta de coherencia entre lo que se dice y lo que se hace, sino más bien, desde una perspectiva comprensiva que mire esta relación complejamente, entendiendo las racionalidades diferentes que caracterizan los discursos respecto a las prácticas.

Tomando en cuenta lo anterior, surgen interrogantes con relación a cuáles son los factores que pueden favorecer la comprensión del porqué se proporcionan determinadas ODA. Por este motivo, en el presente estudio se considera relevante profundizar en el análisis de las creencias del profesorado que orientan las decisiones pedagógicas y guían el proceso de aprendizaje (Ahsan, Sharma y Deppeler, 2012; Edwardraj, Mumtaj, Prasad, Kuruvilla y Jacob, 2010).

Existe evidencia que indica que las creencias sobre el aprendizaje y la enseñanza afectan las prácticas del profesorado (Porter, 1991; Thompson, 1992). En el desarrollo teórico de las creencias referidas a las matemáticas, se han identificado tres dimensiones: creencias sobre las matemáticas 
cómo objeto de estudio, creencias sobre la naturaleza de la enseñanza de las matemáticas, y creencias respecto al aprendizaje de las matemáticas (Ernest, 1991; Schmidt y McKnight, 1995). En esta línea, la investigación se ha desarrollado por medio del análisis de observaciones de aula, de los materiales curriculares y de las prácticas declaradas por los docentes (Polly y Hannafin, 2011). No obstante, se carece de investigaciones similares en estudiantes en situación de DI.

En resumen, en Chile no contamos con estudios que muestren las ODA que tienen los estudiantes con Dı en la enseñanza de las matemáticas. De este modo, el objetivo de la presente investigación es analizar cuáles son las creencias y prácticas declaradas respecto a las matemáticas y su proceso de enseñanza y aprendizaje con estudiantes que presentan DI, para de este modo explorar y comprender las ODA que se generan en escuelas especiales.

\section{Metodología}

Este artículo reporta una sección de los resultados de una investigación más amplia que utilizó un diseño de estudio de casos múltiples (Yin, 1994) por medio de tres componentes: entrevistas semiestructuradas (Canales, 2006; Taylor y Bogdan, 1984), registro de material de aula y observación de clases. El estudio de casos no busca lograr una representatividad en sus hallazgos, sino que busca la generalidad en su relación con la teoría.

Aquí se dan a conocer los resultados relacionados con el objetivo de explorar las razones y los criterios que orientan las decisiones del profesorado respecto a la implementación, flexibilización y adecuación del currículo de matemáticas para estudiantes con Dı. Específicamente, se reportan los resultados de las entrevistas desarrolladas con nueve profesores de educación especial, tres de los cuales se desempeñan como directores, tres como coordinadores técnico-pedagógicos (UTP) ${ }^{2}$ y tres como profesoras de cursos de primer ciclo básico (Básico 5 y Básico 7) ${ }^{3}$ de tres escuelas especiales, de la ciudad de Santiago de Chile. En el desarrollo de las entrevistas se profundizó en la descripción de los procesos de diseño, desarrollo y evaluación de sus clases y del aprendizaje de sus estudiantes, así como en los significados que ellos les atribuyen a las matemáticas, a su enseñanza y aprendizaje, particularmente con estudiantes con DI.

2 Los coordinadores técnico-pedagógicos en chile son conocidos como utp dado que dirigen la Unidad Técnico Pedagógica.

3 Los cursos de escuelas especiales se rigen actualmente por el Decreto 87 de 1990, que aprueba los planes y programas de estudio para personas con discapacidad intelectual. La edad de los estudiantes de los cursos Básico 5, 6 y 7 puede variar entre los 8 y 11 años de edad. (http://portales.mineduc.cl/usuarios/edu.especial/doc/201304231709370. DecretoN87.pdf). 
Una vez que se transcribieron las entrevistas, se realizó un análisis de contenido por medio de la aplicación sistemática del método comparativo constante, los criterios de muestreo teórico y la saturación conceptual de las categorías y subcategorías emergentes (Glaser y Strauss, 2009). De este modo, para la reducción de los datos se realizaron procesos de codificación y categorización. Se empezó con una codificación abierta seguida de una codificación axial, lo que permitió definir categorías y subcategorías, así como sus relaciones para, de este modo, comprender el fenómeno en estudio y responder a la pregunta referida a cuáles son las creencias de los profesores y directivos de estudiantes con DI respecto de la enseñanza, de los alumnos y de las matemáticas.

En cuanto a los aspectos éticos, este estudio protegió los derechos, la privacidad y el bienestar de las personas involucradas, siguiendo los principios de resguardo ético. Para tal fin, antes de la entrevista cada participante firmó un consentimiento informado que aseguraba conocimiento de la investigación, confidencialidad y voluntariedad de su intervención.

\section{Resultados}

El análisis de los datos permitió configurar una categoría central referida a las creencias y prácticas declaradas respecto a la enseñanza de las matemáticas, de la cual se desprenden cuatro subcategorías. Por medio de estas, es posible describir y analizar las ODA que se generan en escuelas especiales para estudiantes con DI.

La presentación de los resultados se organiza en cinco ámbitos: 1) la forma en que se concibe la enseñanza de las matemáticas para estos estudiantes en escuelas especiales y la relación con elementos curriculares, 2) la planificación de la enseñanza, 3) las metodologías de enseñanza, 4) los recursos para la enseñanza, y 5) los principales elementos referidos a la evaluación de aprendizajes. Para cada ámbito se ofrece una descripción de lo que emerge desde los actores, así como relatos que lo ejemplifican. Estos resultados se pueden observar sintéticamente en la figura 1. 


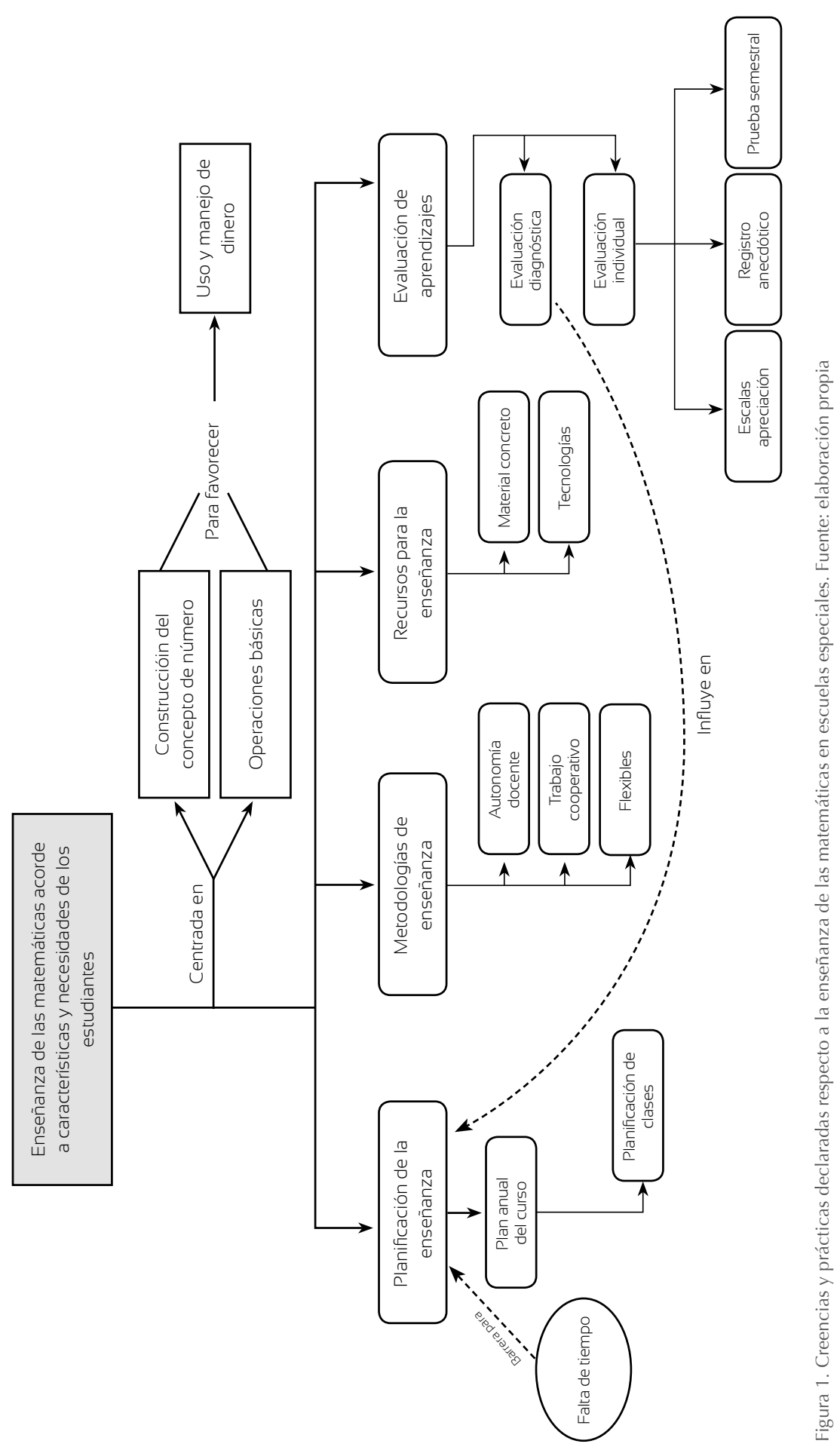


A partir de las respuestas de los entrevistados, se constató que un elemento generalizado y compartido por ellos es la forma en que se conceptualiza la enseñanza de las matemáticas, la que se debe adecuar y ajustar en función de las características y necesidades de los estudiantes que asisten a la escuela especial. De este modo, persiste la creencia de que la manera de responder a las diferentes necesidades educativas de los estudiantes es por medio de una flexibilidad que permita hacer frente a las situaciones específicas que se dan en el aula y por medio de actividades acordes con el potencial de aprendizaje de los estudiantes.

Asimismo, se configura una categoría central a partir de la cual se sostiene que la diversificación de la enseñanza de las matemáticas es clave para una enseñanza equitativa que responda a las características y necesidades de los estudiantes.

\section{Enseñanza de las matemáticas centrada en la construcción del concepto de número y del aprendizaje de las operaciones básicas}

Desde las creencias y prácticas declaradas de docentes y directivos, las matemáticas que deben estudiar los alumnos con Dı están relacionadas principalmente con la construcción del concepto de número. Por esta razón, opinan que es necesario trabajar algunas habilidades previas consideradas indispensables para esto, tal como se observa a continuación:

... ahora empezamos los números... como para que empiecen a acercarse, pero todavía estamos afianzando lo que es la clasificación, la correspondencia y la seriación. (Profesora 2).

... porque dentro de los contenidos de matemáticas nosotros nos enfocamos solamente al tema de las operaciones básicas y numerales. (Profesora 1).

De acuerdo con sus parámetros, y como veremos a partir de los resultados de las evaluaciones diagnósticas, los profesores seleccionan el ámbito numérico y su gradualidad en el plan anual:

Se hizo el plan anual y según el plan anual se va trabajando cada mes, por ejemplo en abril nos tocó en primero básico del 0 al 9, si no me equivoco, 0 al 9 en ma... abril, en mayo nos tocaron los números ordinales eso es en primero, en segundo en abril nos tocaba del 20 al 40 si no me equivoco. (Profesora 1).

Un aspecto recurrente en las creencias y prácticas declaradas de los entrevistados se refiere a que el desarrollo de los conceptos de número y de las operaciones básicas apunta al desarrollo del uso y manejo del dinero: 
Todavía no he querido meterme con nada con el dinero. He tratado de ir pausada... procurando que no queden vacíos porque esos vacíos después pesan, si el chiquillo no comprendió bien el concepto número, si no logró reconocer la secuencia numérica completa, eso ya después el próximo año y el siguiente les va a pesar. (Profesora 2).

De este modo, los entrevistados entienden que el uso del dinero es una práctica que los estudiantes deben desarrollar:

... el tema del concepto del dinero, a través de cosas concretas como ir a la feria (a comprar) hasta cosas de... o al supermercado donde sea necesario para que vayan viendo los valores, hacer listas, precios, todo lo demás. (Director 3).

\section{Planificación de la enseñanza}

El proceso de enseñanza y aprendizaje de las matemáticas se planifica de acuerdo a las decisiones de cada docente. De este modo, los profesores de escuelas especiales tienen la libertad de seleccionar los objetivos de aprendizaje para sus estudiantes, ya que -hasta la fecha- estas escuelas en Chile no tienen la obligación legal de regirse por las bases curriculares nacionales.

El criterio de selección de los aprendizajes de matemáticas evidenciado por los participantes es principalmente el resultado de la evaluación diagnóstica que realizan al inicio del año escolar. Esta es de carácter formativo, y en consonancia con ella se diseña un plan anual para la enseñanza de las matemáticas, es decir, se seleccionan las matemáticas que se les enseñarán de acuerdo al nivel de competencias de los estudiantes:

... entonces tenemos el proceso de evaluación diagnóstica la cual [sic] se desarrollan los planes anuales de trabajo por asignatura, y de ahí, se desprenden las planificaciones mensuales y diarias. (UTP 3 ).

Este plan educativo es general para el curso, por tanto, las necesidades educativas especiales de los estudiantes se abordan a nivel curricular a través de su flexibilización en las planificaciones mensuales que los profesores realizan y en la puesta en práctica de la actividad en el aula:

... entonces por ejemplo si hay que trabajar con números, siempre la planificación se hace en dos grupos, grupo 1 que es el de primero, numerales del 0 al 9 por ejemplo y grupo 2 del 20 al 40 por ejemplo. Ahora en la práctica se hacen como las clases expositivas, en el inicio de la clase lluvia de ideas se expone el tema, por ejemplo un power o... con algún tipo de estímulo audiovisual, videos los típicos como estos que son muy infantilizados, pero en realidad a los chiquillos les gusta y después ya empezamos a trabajar firme [sic]. (Profesora 1). 
Las adecuaciones curriculares se abordan en los planes de estudio especiales elaborados por la escuela:

... una vez realizado el plan anual de trabajo que está hecho absolutamente en base a [sic] las asignaturas... y a los contenidos y aprendizajes esperados del currículum nacional que obviamente tiene, tienen adecuaciones también porque a veces los aprendizajes esperados son muy amplios, por lo cual los profesores realizan la adecuación sin perder la esencia del Aprendizaje Esperado que es lo importante. (UTP 3).

Sin embargo, para los casos en que se requiere de una mayor flexibilización curricular esta no se aplica por la escasez de tiempo para reunir al equipo de profesionales:

... [la implementación de las adecuaciones curriculares] es solo a nivel de, de cómo la evaluación de la Jefa Técnica a... a los profesores, pero, así como que dejen registrado, que esta es la adecuación. (Director 1).

Para la selección de los objetivos de aprendizaje y la planificación de las clases se realizan adecuaciones referidas al nivel de complejidad de dichos objetivos:

Filtrando. Ya fracciones, ok, fracciones yo puedo trabajar fracciones, pero enteros medios, cuartos, con suerte el octavo, por qué, porque hay cosas que vienen en un octavo, pero en una torta, en una receta, etcétera. Pero nada de sumar fracciones. (UTP 1).

A partir de los testimonios de los profesores se identifican dos barreras principales que afectan el desarrollo de planificaciones diversificadas acordes a las características y necesidades de los estudiantes, así como a la implementación o desarrollo de lo planificado. Estas barreras son la falta de tiempo no lectivo y las características singulares que cada condición y estudiante muestra, que se interponen a la implementación planificada. Por ejemplo, se pueden mencionar conductas desafiantes, estrategias deficientes para el afrontamiento socio-emocional que el desafío del aprendizaje de las matemáticas plantea, escasas habilidades de comunicación, contextos de alta vulnerabilidad que se interponen al proceso de aprendizaje y/o entre los modelos de resolución de conductas disruptivas que la familia promueve con aquellos propuestos por el espacio educativo.

Los docentes plantean que la falta de tiempo no permite diversificar las planificaciones, aspecto importante, según ellos, frente a las necesidades de los estudiantes. Los profesores afirman que en muchas ocasiones terminan planificando lo mismo y que durante la clase adecuan las actividades: "... pásame una clase te la voy a hacer. Pero no me pidas que planifique, porque la verdad que yo planifico así a la rápida, porque planifico en mi casa" (Profesora 3). 
Otra de las profesoras también indica que planifica fuera de su horario laboral: "... trato en lo posible que sea dentro del horario laboral, pero si pudiera trabajaría hasta las tres. No, me hace igual falta el horario técnico" (Profesora 2).

La falta de tiempo para planificación también es indicada por directivos docentes:

... porque para planificar algo así tú tienes que planificar de manera pensada, y pensar la planificación demanda tiempo y creo que los profesores hoy por hoy es lo que menos tiempo o piensa en la planificación lo que te va a dar también respuesta en las estrategias que vas a utilizar, materiales que vas a utilizar. (UTP 2).

El proceso de apoyo y asesoría técnico-pedagógica de entrega de los lineamientos de directivos docentes a profesores para la elaboración de planificación también se ve afectado por la escasez de tiempo:

... mira yo y te digo sinceramente, no me alcanza el tiempo y qué hago yo, focalizó en algunos casos... inclusive en algunas ocasiones lo revisamos en conjunto no presencial, sino a través de correos y focalizo algunos cursos que principalmente aquellos que vienen ingresando a la institución, a la escuela. (UTP 2).

Según se indicó, los avances son lentos y los aprendizajes seleccionados son mínimos en relación con el currículo nacional. Además, los entrevistados concuerdan en plantear que difícilmente se logra cumplir con lo planificado pues inciden factores conductuales en el aprendizaje de los estudiantes:

... a veces igual la planificación no se ha podido llevar a cabo de manera completa, porque hay algún tipo de crisis conductual que un niño se escapó por ejemplo, que quiso seguir jugando con la pelota cosa que el tiempo ya era de estar trabajando en el cuaderno por ejemplo, entonces ahí se nos escapa un poco el tema, pero siempre se trata de finalizar la actividad. (Profesora 1).

Las ideas presentadas por los profesores permiten evidenciar que las actividades especiales, las estrategias y los recursos que desarrollan en el aula responden a las dificultades y necesidades de cada estudiante:

... los objetivos del NT1 él los puede alcanzar, pero a lo mejor se va a demorar más que el resto, entonces se va priorizando con él lo que vamos trabajando, entonces y también a nivel de exigencia va a lo que él nos va mostrando también. (Profesora 2).

Esto se hace más patente en casos excepcionales: “... cuando son casos muy excepcionales por ejemplo algunos niños con trastorno motor, se hace algo como una adecuación diferente una adecuación curricular" (Director 3). 


\section{Metodologías de enseñanza}

En cuanto a las metodologías utilizadas para el aprendizaje de las matemáticas, los directores y responsables de la Unidad Técnico-Pedagógica expresan una falta de lineamientos metodológicos compartidos entre los distintos profesores, por lo cual se evidencia una autonomía docente que se espera sea coherente con las características de los estudiantes, tal como se observa en los siguientes testimonios:

... cada uno trabaja dependiendo de su propio conocimiento, las características de su curso, no hay un lineamiento a nivel de escuela.

(Director 1).

... lineamiento no... claro no hay porque la diversidad de $\operatorname{cabros}^{4}$ no te lo permite, o sea yo soy un poco de la idea de ocupar las técnicas que el cabro necesite... creo que es mejor juntar algunas técnicas. (Director 2)

Por otro lado, los profesores de aula entrevistados establecen una relación entre lo que entienden por metodología con el tipo de organización del aula, centrada específicamente en formas de trabajo cooperativo entre estudiantes: "... también desarrollamos harto lo que es el trabajo colaborativo entre los compañeros... el que terminó antes tiene que ir a ayudar al compañero o el que está por ejemplo segundo, ayuda al que está en primero" (Profesora 1).

Con relación a cómo se trabajan los aprendizajes de las matemáticas en el aula, los actores concuerdan en que las características que presentan estos estudiantes son determinantes para la toma de decisiones y el abordaje de la enseñanza en el aula: "...cómo se ha ido desarrollando el trabajo es más en el que hacer propio de la sala de clases... y también debido a las características de déficit atencional de los niños" (Director 3).

En cuanto a la implementación de estas metodologías para la enseñanza de las matemáticas, un elemento recurrente señalado por los entrevistados se refiere a la variedad y flexibilidad de metodologías y de estrategias. Así, sus prácticas declaradas se refieren al juego como un aspecto central que convive con estrategias más tradicionales de enseñanza "... en la metodología, bueno hemos trabajado harto en lo que es el juego dirigido" (Profesora 2).

Los entrevistados creen que cada estudiante es único, con necesidades particulares, lo que obliga a diversificar el trabajo dependiendo de ellos, pero de lo concreto a lo abstracto: "... debido a las características de déficit atencional de los niños etc., donde lo que se plantea son una diversidad de actividades que vayan diseñadas desde lo más concreto a lo más... gráfico dentro del mismo ciclo de aprendizaje" (Director 3).

4 En Chile, en lenguaje coloquial, cabro se usa como sinónimo de niño. 


\section{Recursos para la enseñanza}

Respecto de los recursos utilizados para el aprendizaje de las matemáticas se destacan dos aspectos centrales: el uso de material concreto y de tecnologías.

Las profesoras de aula expresan que para el desarrollo de las actividades de matemáticas se utiliza una variedad de material concreto, por ejemplo, recursos naturales y objetos de desecho que se reciclan para crear materiales de trabajo para el aula:

... en la sala... con piedras hemos trabajado, con bandejas de estas de aislapol que viene como la carne [sic], para que ellos puedan ir asociando la cantidad. (Profesora 1).

... estamos inventando material para trabajar el tema de las matemáticas. (Profesora 2).

Respecto de otros recursos didácticos, los directivos no hacen una alusión precisa de cómo los utilizan en el aula: "... trabajan también con juegos de supermercados, tenemos los dineros, los billetes, las típicas cosas que nosotros trabajamos y también hay muchos juegos en relacionados con... los números, bingos, etc. O sea harto" (Director 3).

Sin embargo, estos recursos no se vinculan explícitamente con los objetivos propuestos en el plan de trabajo de las matemáticas: "... bueno la 'Paulina' trabaja harto material concreto ya, la Romina trabaja por rincones, en todas las asignaturas" (UTP 1).

Los resultados indican que para los aprendizajes relacionados con ámbito numérico y operatoria se trabaja principalmente a través de actividades en la pizarra, que los estudiantes copian en sus cuadernos o bien que desarrollan en guías de trabajo:

... [usamos] el libro y el cuaderno, porque es una situación de que yo soy grande. Yo trabajo con libro y con cuaderno. (Educadora 3).

... [la profesora] trabaja con cuaderno, trabaja con guía, con lámina, también trabaja en forma lúdica. (UTP 3).

Por otra parte, los directivos hacen alusión principalmente a los recursos tecnológicos con que cuentan las escuelas para la enseñanza y aprendizaje de las matemáticas: “... en este momento la escuela tiene algunas TIC; hay una, hay una pizarra interactiva" (Director 1).

Los entrevistados manifiestan que las TIC son un recurso usado principalmente como apoyo audiovisual, a través de presentaciones y del trabajo con actividades interactivas de matemáticas que desarrollan en el computador: 
... el data lo he usado harto también, la verdad es que nos hemos estado aprendiendo una canción de los números por ejemplo. (Profesora 2).

... ocupamos mucho computador de hecho en la sala tenemos 4 computadores, se le manda material a los chiquillos, cada uno de ellos tiene un prendive [sic], se les manda por ejemplo si en la semana se trabaja los números ordinales, se le manda un video correspondiente a los números ordinales y lo trabajan desde su casa también. (Profesora 1).

\section{Evaluación de aprendizajes}

En el contexto de las escuelas especiales, la evaluación de aprendizajes para estudiantes con DI se inicia con una evaluación diagnóstica, a partir de la cual se toman decisiones para planificar la enseñanza. Dicho de otro modo, esta evaluación permite el diseño del plan anual y define el alcance de los planes individuales. Se lleva a cabo preferentemente por medio de procedimientos cualitativos (registro anecdótico, escalas de apreciación) y en menor medida en evaluaciones sumativas semestrales, mediante procedimientos cuantitativos.

La evaluación inicial permite conocer los aprendizajes previos de los estudiantes. Para ese diagnóstico los profesores pueden apoyarse en los resultados obtenidos en las evaluaciones que desarrolla la institución en la que enseñan, o pueden aplicar evaluaciones diagnósticas con instrumentos construidos por ellos:

... dentro de lo que es las matemáticas nosotros tenemos diferentes formas de evaluar, primero que lo hacemos en el proceso de marzo, tenemos la evaluación que nos manda la Fundación... pero aparte de eso nosotros manejamos pruebas que nosotros hemos diseñado dentro del colegio. (Director 3).

El proceso de evaluación se presenta con escasa estructura y lineamientos que permitan señalar con claridad cómo evaluar, qué instrumentos aplicar y cómo transformar en una calificación los datos cualitativos que obtienen de los instrumentos de observación:

... nosotros hacemos evaluaciones mensuales, que son escalas de apreciaciones según las observaciones que nosotros hacemos en clases... por ejemplo en abril se trabajó del 0 al 9 se evalúa con una prueba que puede ser de alternativa o de desarrollo, aparte igual enviamos tareas para la casa. (Profesora 1).

... para lo que soy buena es para el registro anecdótico de las observaciones directas, voy anotando todo, entonces eso quiero ver como lo plasmo a nivel de evaluación cualitativa como para apoyar un poco $[\ldots]$ en el fondo tener claridad de hasta dónde llegamos y qué es lo que podemos seguir haciendo con ellos y eso queda mejor 
plasmado a nivel cualitativo que cuantitativo, que en un número o en una letra. (Profesora 2).

Para los entrevistados, el proceso de evaluación debe contemplar una etapa que se realiza de manera individual, contemplando las características de cada estudiante: "... uno a uno. Hay unos que se les olvidó pasar la prueba, otros que no" (Educadora 2).

Con instrumentos variados: "es variado. Pero yo diría que la gran parte... a nivel de observación, más que pruebas específicas de... un instrumento informal, es más bien observación directa y se va estableciendo la evaluación de, de los cursos" (Director 1).

\section{Conclusiones}

El estudio exploró y analizó las creencias y prácticas declaradas de los profesores de aula y de equipos directivos de escuelas especiales, respecto a las matemáticas y a su proceso de enseñanza y aprendizaje. Para ello se siguió un enfoque cualitativo descriptivo, por lo que no se pretende generalizar los hallazgos aquí presentados, sino comprender desde las experiencias singulares aspectos centrales de dichas creencias y prácticas declaradas.

Los resultados indican que: 1) la enseñanza de las matemáticas, en estos contextos educativos, se concibe como un proceso que debe ajustarse a las características y necesidades de los estudiantes, más que a las exigencias curriculares; 2) la enseñanza se centra en el aprendizaje de los números y de las operaciones básicas, pues se concibe que son elementos clave para el uso del dinero en la comunidad; 3) la planificación de la enseñanza se nutre de evaluaciones diagnósticas que facilitan el diseño de planes anuales de curso y su posterior ajuste a casos particulares de estudiantes, sin embargo, la falta de tiempo no lectivo es una barrera para estos procesos de diseño; 4) la evaluación del aprendizaje se sustenta en una mirada individual de este proceso, que se lleva a cabo por medio de instrumentos cualitativos y cuantitativos para medir resultados de aprendizaje semestrales. Con un énfasis menor, los resultados también indican que las metodologías de enseñanza son flexibles y contemplan estrategias lúdicas así como otras más tradicionales e individuales, como el trabajo en cuadernos, libros de texto y guías de trabajo y que, en el desarrollo de las clases de matemáticas, se utilizan recursos tecnológicos y material concreto que busca facilitar la manipulación y el conteo.

Esta evidencia da cuenta de creencias y prácticas educativas sustentadas en el desarrollo de procesos de aprendizaje basados en un currículo restringido y menos riguroso (Nolet y McLaughlin, 2000), con un uso intensivo de materiales concretos (Sarama y Clements, 2009); y con la necesidad de realizar adecuaciones curriculares individuales (Stainback y Stainback, 
1999). Si bien en ocasiones es necesario hacer ajustes individuales, esta postura de visualizar el proceso de enseñanza caso a caso podría responder a una lógica de trabajo extendida en la enseñanza especial de corte cognitivista (Woolfolk, 2011), focalizando las habilidades particulares, pero sin acceder a otras perspectivas que entienden que la enseñanza y el aprendizaje ocurren como una co-construcción de todos los participantes.

Los resultados dan cuenta de una limitación de las ODA en matemáticas que se ofrecen a estudiantes con DI y, con ello, de la equidad educativa, específicamente restringiendo las matemáticas al concepto de número asociado al uso del dinero. Llama la atención en las creencias y prácticas declaradas por los profesores y directivos, la ausencia de las habilidades matemáticas. Ellas cumplen, como componente transversal, un rol articulador que permitiría el desarrollo de los estudiantes en cuestiones como la argumentación, la representación y el modelamiento.

Este escenario promueve una serie de desafíos para la formación docente en matemáticas y educación diferencial. Por una parte, es fundamental que los programas universitarios de formación de profesores y de formación continua incorporen cursos referidos a currículo y enseñanza de las matemáticas desde modelos centrados en las habilidades. Por otra parte, se requiere incorporar en estos procesos estrategias pedagógicas que permitan diversificar la enseñanza para todos los estudiantes, como por ejemplo el diseño universal de aprendizaje (Spooner, Baker, Harris Ahlgrim-Delzell y Browder, 2007).

En cuanto a las barreras asociadas al tiempo no lectivo de los profesores y su incidencia en el diseño y la planificación del proceso de enseñanza, los resultados permiten señalar que se requieren transformaciones en la organización interna de las escuelas especiales, pero sobre todo en las políticas educativas nacionales, que hagan posibles esas transformaciones.

Finalmente, es necesario precisar que se deben desarrollar futuras investigaciones que complementen los resultados aquí expuestos, con el propósito de generar orientaciones para la práctica educativa en matemáticas con estudiantes con DI en primer ciclo básico. Con todo ello, se espera avanzar hacia un verdadero acceso equitativo a las ODA en matemáticas, sustentado en un enfoque de derecho a la educación de calidad para todos los estudiantes.

\section{Referencias}

Agut, N. (2010). La evaluación en un modelo de escuela inclusiva. Aula de Innovación Educativa, 191, 42-44.

Ahsan, M. T., Sharma, U. y Deppeler, J. M. (2012). Exploring pre-service teachers' perceived teaching-efficacy, attitudes and concerns about inclusive education in Bangladesh. International Journal of Whole Schooling, 8(2), 1-20. 
Ainscow, M. (2001). Necesidades especiales en el aula: Guía para la formación del profesorado. Madrid: Narcea.

Ainscow, M.; Booth, T. y Dyson, A. (2006). Improving schools developing inclusion. Londres: Routledge.

Banicky, L. (2000). Opportunity to learn. Education Policy Brief, Delaware 7, 1-4.

Bishop, A. y Forgasz, H. (2007). Issues in access and equity in mathematics education. En F. Lester (ed.). Second handbook of research on mathematics teaching and learning (vol. 2, pp. 1145-1167). Reston: NCTM

Blanco, R. (1999). Hacia una escuela para todos y con todos. Boletín Proyecto Principal de Educación en América Latina y el Caribe. N 48, abril de 1999, unESCO/Orealc, Santiago de Chile.

Boscardin, C.; Aguirre-Munoz, Z.; Stoker, G.; Kim, J.; Kim, M. y Lee, J. (2005). Relationship between opportunity to learn and student performance on English and Algebra assessments. Educational Assessment, 19(4), 307-332. DOI: 10.1207/s15326977ea1004_1

Browder, D. M.; Trela, K.; Courtade, G. R.; Jimenez, B. A.; Knight, V. y Flowers, C. (2012). Teaching mathematics and science standards to students with moderate and severe developmental disabilities. The Journal of Special Education, 46, 26-35. Dol:10.1177/0022466910369942

Carrillo, J.; Contreras, L. y Zakaryan, D. (2013). Avance de un modelo de relaciones entre las oportunidades de aprendizaje y la competencia matemática. Boletim de Educação Matemática, 27(47), 779-804.

Cawley, J. F. y Miller, J. H. (1989). Cross-sectional comparisons of the mathematics performance of children with learning disabilities: Are we on the right track towards comprehensive programming? Journal of Learning Disabilities, 22, 250-254.

Cheong, J. (2014). Numeracy learning of students with mild intellectual disabilities in Singapor (tesis de maestría). National Institute of Education, Nanyang Technological University.

Cogan, L. S., y Schmidt, W. H. (2015). The concept of Opportunity to Learn (OTL) in International Comparisons of Education. En Assessing Mathematical Literacy (pp. 207-216). Springer International Publishing.

Duk, C. y Loren, C. (2010). Flexibilización del currículum para atender la diversidad. Revista Latinoamericana de Inclusión Educativa, 4(1), 187-210.

Edwardraj, S.; Mumtaj, K.; Prasad, J. H.; Kuruvilla, A. y Jacob, K. S. (2010). Perceptions about intellectual disability: A qualitative study from Vellore. Journal of Intellectual Disability Research, 54(8), 736-748.

Ernest, P. (1991). The impact of beliefs on the teaching of mathematics. En P. Ernest (ed.), Mathematics teaching: The state of the art (pp. 249254). Londres: Falmer. 
Faragher, R.; Brady, J.; Clarke, B. y Gervasoni, A. (2008). Children with Down Syndrom learning mathematics: Can they do it? Yes they can! Australian Primary Mathematics Classroom, 13(4), 10-15.

Ferrer, M.; Fortuny, J. M. y Morera, L. (2014). Efectos de la actuación docente en la generación de oportunidades de aprendizaje matemático. Enseñanza de las Ciencias, 32(3), 385-405.

Forgasz, H. y Rivera, F. (2012). Towards equity in mathematics education. Berlín: Springer.

Gallico, R.; Burns, T. J. y Grob, S. C. (1991). Emotional and behavioral problems in children with learning disabilities. San Diego, CA: Singular.

Gervasoni, A. y Lindenskov, L. (2011). Students with "special rights" for mathematics education. En B. Atweh, M. Graven y W. Secada (eds.). Mapping Equity and Quality in Mathematics Education, pp. 307323. Netherlands: Springer.

Gervasoni, A. y Sullivan, P. (2007). Assessing and teaching children who have difficulty learning arithmetic. Educational \& Child Psychology, 24(2), 40-53.

Gil, N.; Blanco, L. y Guerrero, E. (2005). El dominio afectivo en el aprendizaje de las matemáticas. Una recisión de sus descriptores básicos. Revista Iberoamericana de Educación Matemática, 2, 15-32.

Glaser, B. G., y Strauss, A. L. (2009). The discovery of grounded theory: Strategies for qualitative research. New Jersey: Transaction.

Gómez-Chacón, I. M. (2003). La tarea intelectual en matemáticas. Afecto, meta-afecto y los sistemas de creencias. Boletín de la Asociación Matemática Venezolana, 10(2), 225-247.

Hiebert, J. y Grouws, D. A. (2007). The effects of classroom mathematics teaching on students' learning. Second handbook of research on mathematics teaching and learning, 1, 371-404.

Jimenez, B. A., Browder, D. M. y Courtade, G. R. (2008). Teaching algebra to students with moderate cognitive disabilities. Education and Training in Developmental Disabilities, 43, 266-274.

Jimenez, B. y Staples, K. (2015). Access to the Common Core State Standards in mathematics through early numeracy skill building for students with significant intellectual disability. Education and Training in Autism and Developmental Disabilities, 50(1), 17-30.

Kauffman, J. M. (2001). Characteristics of emotional and behavioral disorders of children and youth (7. ${ }^{\text {a }}$ ed.). Upper Saddle River, NJ: Simon y Schuster.

Kearns, J. F.; Towles-Reeves, E.; Kleinert, H. L.; Kleinert, J. O. y Thomas, M. K. (2011). Characteristics of and implications for students participating in alternate assessments based on alternate academic achievement standards. The Journal of Special Education, 45, 3-14. 
Martín, E. y Mauri, T. (1996). La atención a la diversidad como eje vertebrador de la educación secundaria. En E. Martín y T. Mauri (coords). La atención a la diversidad en la educación secundaria (pp. 13-36). Barcelona: ICE/Horsori.

Martínez, O. (2013). Las creencias en la educación matemática. Educere, 18(57), 231-239.

McDonnell, L. M. (1995). Opportunity to learn as a research concept and policy instrument. Educational Evaluation and Policy Analysis, 17(3), 305-322.

Ministerio de Educación Nacional. (2004). Implementación curricular en el aula. Matemáticas Primer Ciclo Básico. Seguimiento a la implementación curricular. Unidad, currículum y evaluación.

Nolet, V., y McLaughlin, M. J. (2000). Accessing the general curriculum: Including students with disabilities in standards-based reform. Thousand Oaks, CA: Corwin Press

Organización de las Naciones Unidas para la Educación, la Ciencia y la Cultura,unESCO. (2009). Directrices sobre políticas de inclusión en educación.

Organización Mundial de la Salud (2001). Clasificación internacional del funcionamiento de la discapacidad y la salud: CIF. Edición en español, Instituto de Migraciones y Servicios Sociales, Imserso. Madrid, España.

Polly, D. y Hannafin, M. J. (2011). Examining how learner-centered professional development influences teachers' espoused and enacted practices. Journal of Educational Research, 104, 120-130.

Porter, A. (2002). Measuring the content of instruction: Uses in research and practice. Educational research, 31(7), 3-14.

Porter, A. C. (1991). Creating a system of school process indicators. Education and Policy Analysis, 13, 13-29.

Rodrigo, M. J.; Rodríguez, A. y Marrero, J. (1993). Las teorías Implícitas. Una aproximación al conocimiento cotidiano. Madrid: Aprendizaje Visor.

Rojas, M. T. (2014). Las creencias docentes: delimitación del concepto y propuesta para la investigación. Revista Electrónica Diálogos Educativos, 14(27), 89-112.

San Martín, C. (2012). Atención de la diversidad en el contexto educativo chileno: concepciones del profesorado sobre evaluación y diseño de la propuesta curricular. Reice. Revista Iberoamericana sobre Calidad, Eficacia y Cambio en Educación, 10(4), 164-183.

Sarama, J. y Clements, D. H. (2009). Early childhood mathematics education research: Learning trajectories for young children. Nueva York, NY: Routledge.

Schmidt, W. y McKnight, C. (1995). Surveying educational opportunity in mathematics and science: An international perspective. Educational Evaluation and Policy Analysis, 17(3), 337-353. 
Spooner, F.; Baker, J. N.; Harris, A. A.; Ahlgrim-Delzell, L., y Browder, D. M. (2007). Effects of training in universal design for learning on lesson plan development. Remedial and Special Education, 28(2), 108-116.

Stainback, S. y Stainback, W. (1999). Aulas inclusivas. Madrid: Narcea.

Sullivan, P.; Clarke, D.; Clarke, B. y O'Shea, H. (2010). Exploring the relationship between task, teacher actions, and student learning. PNA, 4(4), pp. 133-142.

Taylor S. J. y Bogdan, R. (1984). Introducción a los métodos cualitativos de investigación. Barcelona: Paidós Iberoamérica.

Thompson, A. (1992). Teachers' beliefs and conceptions: a synthesis of the research. En D. Grouws (ed.). Handbook of research on mathematics teaching and learning (pp. 127-146). Nueva York: Macmillan.

Woolfolk, A. (2011). Psicología educativa (11. ${ }^{a}$ ed.). Pearson: México.

Yin, R. (1994). Investigación sobre estudio de casos: diseño y métodos (2. ${ }^{\mathrm{a}}$ ed). Applied social research methods series (vol. 5). Sage. 